\title{
Finally, CTLA4Ig graduates to the clinic
}

\author{
Mohamed H. Sayegh \\ Laboratory of Immunogenetics and Transplantation, Brigham and Women's Hospital, Harvard Medical School, \\ 75 Francis Street, Boston, Massachusetts 02115, USA. Phone: (617) 732-5259; Fax: 617-732-5254; \\ E-mail: msayegh@rics.bwh.harvard.edu.
}

It has long been known that $\mathrm{T}$ cells require two signals for full activation, but the mechanisms of how these signals function have been only recently elucidated (1). The first signal is provided by the $\mathrm{T}$-cell receptor after interacting with the MHC/antigenic peptide complex. This so-called "signal one" confers antigen specificity to the immune response but alone is insufficient for full T-cell activation. Indeed, $T$ cells receiving only signal one are rendered anergic (unresponsive to antigenic rechallenge, with inhibition of proliferation and cytokine production) in vitro (2). The second signal, or "costimulatory signal," is provided by interactions between specific receptors on the $T$ cell and their respective ligands on antigen-presenting cells (APCs). The CD28/CD152-B7-1/B7-2 T-cell costimulatory pathway is a unique and complex pathway that regulates $\mathrm{T}$-cell activation (recently reviewed in refs. 3 and 4) (Figure 1). Interaction of CD28, constitutively expressed on $T$ cells, with the B7 family of molecules (B7-1 and B7-2), expressed on APCs, provides a second "positive" signal that results in full T-cell activation, including cytokine production, clonal expansion, and prevention of anergy. In addition, CD28 signaling appears to be important in prevention of cell death and promotion of cell survival, presumably by upregulation of T-cell expression of bcl-xl genes (5).

Once activated, $\mathrm{T}$ cells express another costimulatory molecule (CD152, or CTLA4) that is homologous to CD28, has a higher affinity to B7-1 and B7-2, and functions to provide a "negative" signal that inhibits cytokine production and arrests cell cycle progression (6-8). The importance of CTLA4 as a negative regulatory $\mathrm{T}$-cell costimulatory molecule in the physiologic termination of T-cell responses (9) is highlighted by the obser- vation that CTLA4 gene knockout mice develop massive lymphoproliferation and early death $(10,11)$. Furthermore, recent evidence suggests that CTLA4 negative signaling pathway may be required for the induction of acquired tolerance $(12,13)$. Indeed, it has been hypothesized that CTLA4 may function as a "master switch" for peripheral T-cell tolerance in vivo (14).

Several years before the regulatory function of CTLA4 was elucidated, Linsley et al. first described the creation of a new immunomodulatory agent that consists of the extracellular domain of the soluble CTLA4 receptor fused to the heavy chain of human IgG1 (6). Other similar agents have been subsequently described, including a murine form of CTLA4Ig, and several hundred articles have been published describing the immunomodulatory functions of CTLA4Ig in several experimental animal models of transplant

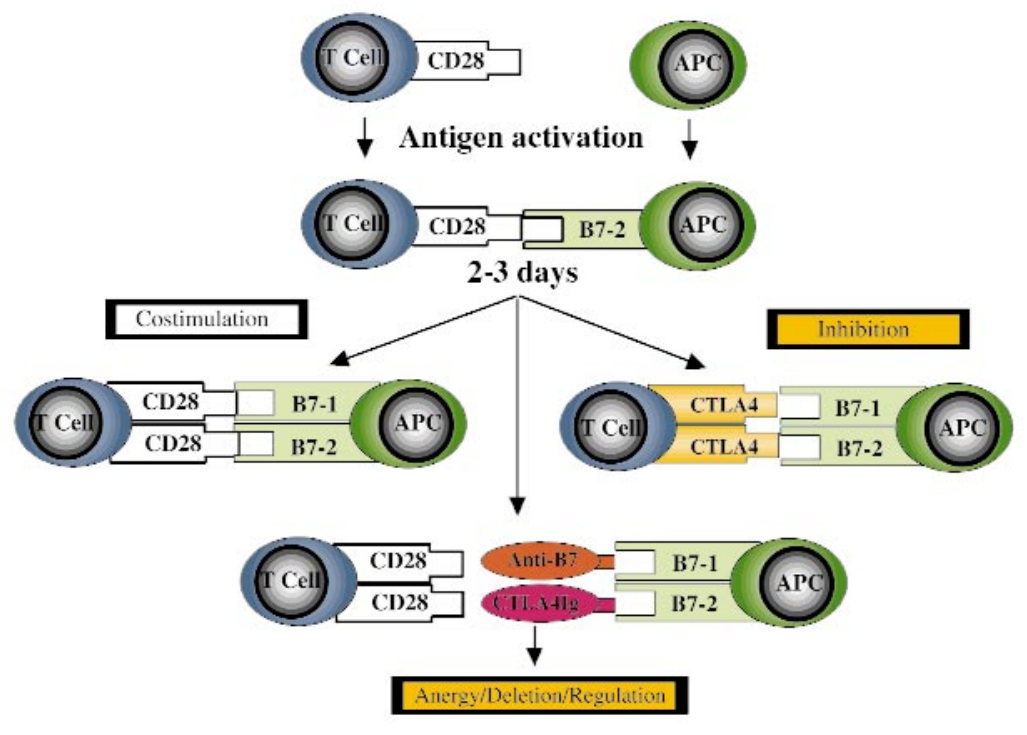

Figure 1

Complexity of the CD28/CD152-B7-1/B7-2 T-cell costimulatory pathway. After antigenic stimulation (delivery of signal one through the T-cell receptor; not shown here), CD28, expressed on resting T cells, interacts with B7-2, and later with B7-1, both expressed on APCs. This results in transduction of a positive costimulatory signal to the $T$ cell, culminating in cytokine production, clonal expansion, and prevention of anergy and cell death, thus promoting cell survival. Activated T cells then express CTLA4, a molecule that is highly homologous to CD28 but upon interaction with B7-1/ B7-2 delivers a negative signal to the T cell, resulting in inhibition of cytokine production and cell cycle progression arrest, thus physiologically terminating immune responses. The use of biologic agents, such as anti-B7 monoclonal antibodies or CTLA4Ig, to block B7 binding to CD28 results in T-cell anergy in vitro, and in anergy, deletion, or induction of regulatory T cells in vivo (4). 
Table 1

Human diseases in which CD28/B7 T-cell costimulatory blockade may have promise

\begin{tabular}{cc}
\hline Autoimmune diseases & Transplant rejection \\
Psoriasis & Solid organs \\
Rheumatoid arthritis & Cell transplants (islets, neural cells) \\
Multiple sclerosis & Bone marrow \\
Systemic lupus erythematosus & \\
Inflammatory bowel diseases & \\
\hline
\end{tabular}

rejection, autoimmunity, infections, asthma, and others (recently reviewed in refs. 3 and 4). Although it is clear that CTLA4Ig, because of the higher affinity of the CTLA4 receptor to B7-1 and B72 , acts as a competitive inhibitor of CD28-B7-1/B7-2 costimulation and induces T-cell anergy in vitro, its exact mechanism of action in vivo remains unclear. It has been suggested, however, that induction of tolerance by B7 blockade may be due to anergy (failure of clonal expansion), deletion, or induction of regulatory $T$ cells in vivo (15-21) (Figure 1). Interestingly, a recent study from our group indicated that an intact CTLA4 negative signaling pathway is required for the immunosuppressive effects of CTLA4Ig in a mouse heart transplant model, adding further to the complexity of the B7-1/B7-2 costimulatory pathway in regulating immune responses (22).

After almost a decade of laboratory studies, CTLA4Ig finally "graduates" to the clinic. In this issue, Abrams et al. (23) present the results of a phase I clinical trial describing the immunosuppressive effects of CTLA4Ig in the T cell-mediated autoimmune skin disease psoriasis vulgaris. This study is unique because it is the first report describing the effects of blocking T-cell costimulatory activation in vivo in human disease. Moreover, although it is difficult to study mechanisms of action of new immunomodulatory therapies in humans, the authors describe the pathologic and immunologic correlates of CTLA4Ig therapy in their patient population. The study showcases the importance of continued T-cell activation in the pathogenesis of psoriasis. Furthermore, although efficacy results of phase I trials should be interpreted with extreme caution, it appears that CTLA4Ig is safe and is at least as effective as conventional therapy for psoriasis in a comparable patient population. However, what is most interesting is the potential for a prolonged beneficial clinical effect of therapy even after CTLA4Ig serum levels become undetectable. These cautionary data in particular suggest that CTLA4Ig may be inducing a state of T-cell hyporesponsiveness or tolerance in vivo.

Two interesting observations in this study again highlight the complexity of the CD28/CD152 T-cell costimulatory signaling pathway. First, there is the dichotomy between the clinical observation indicating that the beneficial effects of CTLA4Ig may be long lasting and the immunologic studies showing that fully primed $\mathrm{T}$ cell-dependent humoral immune responses were not affected, suggesting absence of immunologic tolerance. Second, there is the paradoxical result showing the divergence of suppression of cell-mediated and humoral immune responses at the high-dose schedule $(50 \mathrm{mg} / \mathrm{kg})$ of CTLA4Ig therapy. This latter observation and the recent studies by Judge et al. (22) make one wonder whether in certain diseases, complete blockade of B7-1/B7-2 may not be desirable because it may result in inhibition of a beneficial negative regulatory signal through CTLA4.

Although CTLA4Ig can now celebrate its graduation to the clinic, there is still much to learn. We need to understand in which diseases it is most effective and whether it provides a clear advantage over standard therapies (Table 1). We need to study its safety and efficacy profile in randomized, controlled trials. We need to determine what doses and protocols we should use in patients with different diseases. We need to explore whether CTLA4Ig may be used safely and effectively with other immunosuppressive agents or agents that block other costimulatory pathways, such as the CD40/CD154 pathway (24-26). Indeed, there are experimental data in small animals to indicate that calcineurin inhibitors, such as cyclosporine, may abrogate the immunosuppressive or tolerogenic effects of B7 and/or CD154 blockade $(24,27,28)$. Finally, we need to investigate and better understand the exact mechanisms of costimulatory blockade in vivo in humans with different diseases and to develop surrogate markers to monitor disease activity and response to therapy.

In a recent study, Guinan et al. (29) used CTLA4Ig to anergize alloreactive bone marrow T cells ex vivo to prevent graft-versus-host disease after haploidentical bone marrow transplantation. Such studies, and the pioneering work by Abrams et al. (23), pave the way for the development of new clinical trials that will further examine the immunomodulatory functions of novel agents that block T-cell costimulatory activation in several immune-mediated human diseases. A better understanding of the mechanisms of these novel agents may make the goal of achieving immunologic tolerance in humans elusive no more.

\section{Acknowledgments}

M.H. Sayegh is a recipient of the National Kidney Foundation Clinician Scientist Award. The author would like to thank Charles B. Carpenter, Samia J. Khoury, and Laurence A. Turka for their helpful comments and review of the manuscript.

1. Janeway, C.A., and Bottomly, K. 1994. Signals and signs for lymphocyte responses. Cell. 76:275-285.

2. Schwartz, R.H. 1990. A cell culture model for T lymphocyte clonal anergy. Science. 248:1349-1356.

3. Reiser, H., and Stadecker, M.J. 1996. Costimulatory $\mathrm{B} 7$ molecules in the pathogenesis of infectious and autoimmune diseases. N. Engl. J. Med. 335:1369-1377.

4. Sayegh, M.H., and Turka, L.A. 1998. The role of T cell costimulatory activation in transplant rejection. N. Engl. J. Med. 338:1813-1821.

5. Noel, P.J., Boise, L.H., Green, J.M., and Thompson, C.B. 1996. CD28 costimulation prevents cell death during primary $\mathrm{T}$ cell activation. J. Immunol. 157:636-642.

6. Linsley, P.S., et al. 1991. CTLA-4 is a second receptor for the B cell activation antigen B7. J. Exp. Med. 174:561-569.

7. Walunas, T.L., et al. 1994. CTLA-4 can function as a negative regulator of $\mathrm{T}$ cell activation. Immunity. 1:405-413.

8. Walunas, T.L., Bakker, C.Y., and Bluestone, J.A. 1996. CTLA-4 ligation blocks CD28-dependent T cell activation. J. Exp. Med. 183:2541-2550.

9. Parjis, L.V., and Abbas, A.K. 1998. Homeostasis and self tolerance in the immune system: turning lymphocytes off. Science. 280:243-248.

10. Waterhouse, P., et al. 1995. Lymphoproliferative disorders with early lethality in mice deficient in CTLA4. Science. 270:985-988.

11. Tivol, E.A., et al. 1995. Loss of CTLA-4 leads to massive lymphoproliferation and fatal multiorgan tissue destruction, revealing a critical negative regulatory role of CTLA-4. Immunity. 3:541-547.

12. Perez, V., et al. 1997. Induction of peripheral T cell tolerance in vivo required CTLA-4 engagement. 
Immunity. 6:411-417.

13. Issazadeh, S., Zhang, M., Sayegh, M.H., and Khoury, S.J. 1999. Acquired thymic tolerance: role of CTLA4 in the initiation and maintenance of tolerance in a clinically relevant autoimmune disease model. J. Immunol. 162:761-765.

14. Bluestone, J.A. 1997. Is CTLA-4 a master switch for peripheral $\mathrm{T}$ cell tolerance? J. Immunol. 158:1989-1993.

15. Kearney, E.R., et al. 1995. Antigen-dependent clonal expansion of a trace population of antigen-specific $\mathrm{CD} 4+\mathrm{T}$ cells in vivo is dependent on CD28 costimulation and inhibited by CTLA4. J Immunol. 155:1032-1036.

16. Sayegh, M.H., et al. 1995. CD28-B7 blockade after alloantigenic challenge in vivo inhibits Th1 cytokines but spares Th2. J. Exp. Med. 181:1869-1874.

17. Khoury, S.J., et al. 1995. CD28-B7 costimulatory blockade by CTLA4Ig prevents actively induced experimental autoimmune encephalomyelitis and inhibits Th 1 but spares Th 2 cytokines in the central nervous system. J. Immunol. 155:4521-4524.
18. Judge, T.A., et al. 1996. The in vivo mechanisms of action of CTLA4Ig. J. Immunol. 156:2294-2299.

19. Konieczny, B.T., et al. 1998. IFN-gamma is critical for long-term allograft survival induced by blocking the CD28 and CD40 ligand $\mathrm{T}$ cell costimulation pathways. J. Immunol. 160:2059-2064.

20. Dai, Z., Konieczny, B.T., Baddoura, F.K., and Lakkis, F.G. 1998. Impaired alloantigen-mediated $\mathrm{T}$ cell apoptosis and failure to induce longterm allograft survival in IL-2-deficient mice. J. Immunol. 161:1659-1663.

21. Tran, H.M., et al. 1997. Distinct mechanisms fo the induction and maintenance of allograft tolerance with CTLA4-Fc treatment. J. Immunol. 159:2232-2239.

22. Judge, T.A., et al. 1999. The Role of CD80, CD86 and CTLA4 in alloimmune responses and the induction of long-term allograft survival. J. Immunol. 162:1947-1951.

23. Abrams, J.R., et al. 1999. CTLA4Ig-mediated blockade of T-cell costimulation in patients with psoriasis vulgaris. J. Clin. Invest.103:1243-1252.
24. Larsen, C.P., et al. 1996. Long-term acceptance of skin and cardiac allografts after blocking CD40 and CD28 pathways. Nature. 381:434-438.

25. Kirk, A.D., et al. 1997. CTLA4-Ig and anti-CD40 ligand prevent renal allograft rejection in primates. Proc. Natl. Acad. Sci. USA. 94:8789-8794.

26. Wekerle, T., et al. 1998. Extrathymic T cell deletion and allogeneic stem cell engraftment induced with costimulatory blockade is followed by central T cell tolerance. J. Exp Med. 187:2037-2044

27. Chandraker, A., et al. 1997. T cell costimulatory blockade in experimental chronic cardiac allograft rejection: effects of cyclosporine and donor antigen. Transplantation. 63:1053-1058.

28. Li, Y., Zheng, X.X., Li, X.C., Zand, M.S., and Strom, T.B. 1998. Combined costimulation blockade plus rapamycin but not cyclosporine produces permanent engraftment. Transplantation. 66:1387-1388.

29. Guinan, E.C., et al. 1998. Succesful haploidentical BMT post ex vivo anergization of donor bone marrow alloreactive T cells. Blood. 92:687a (Abstr.) 